\title{
Optical Fiber and its Biomedical Applications: Focusing on Human Heart/Pulse Rate Sensing
}

\author{
Rishi Kant Singh \\ M.Tech Student of ECE Dept. \\ SSET, SHIATS \\ Allahabad.211007, INDIA
}

\author{
A.K.Jaiswal \\ Prof \& HoD of ECE Dept. \\ SSET, SHIATS \\ Allahabad.211007, INDIA
}

\author{
Neelesh Agrawal \\ Assistant Prof. in ECE Dept. \\ SSET, SHIATS \\ Allahabad.211007, INDIA
}

\begin{abstract}
This article focuses on the advantages that optical fiber sensors offer to the biomedical field, the basic working principles of optical fiber sensing, and discusses some examples of integrating different biological parameter fiber sensors on a single probe for Medical Applications. Here our main idea is to initially focus is on the human heart pulse rate monitoring, which includes different $\mathrm{Hb}-\mathrm{O}_{2}$ concentration measurements in one heart beat, using two infrared light sources, and $1 \mathrm{~mm}$ diameter multimode plastic optical fiber as a probe, a photodiode with spectral sensitivity ranging from $300 \mathrm{~nm}-1100 \mathrm{~nm}$ wavelength of light and a open source Arduino Uno R3 interface board for the processing of the incoming signals.
\end{abstract}

\section{Keywords}

Fiber optical sensor, Heart rate, IR LED, Photodiode, Photoplethysmogram, PPG, Pulse oximeter.

\section{INTRODUCTION}

The advances of fluorescence chemistry, lasers technology, optical fiber nano tubes fabrication, development of cheap as well as computationally powerful processors and optical semiconductor technology to solve problems in medical analysis is a fast growing field. This area of applications can be divided as either therapeutic or diagnostic. In therapeutic applications, the Transformation of light energy into chemical, thermal or mechanical energy can cause a direct desired effect or selective cell death (Niemz, 1999). The lighttissue interactions in the diagnostic approach must by contrast be non-destructive and its main goal is to study the physiology or pathology of the tissue which is under investigation. The high potential and safe nature of light as a method to evaluate the light-tissue interactions, such as diffusion, reflection, scattering, polarization, phase, wavelength content and time of flight transmittance among many more is always to be preferred over methods that involve harmful effects under other diagnostic techniques. With this in mind the range from $300 \mathrm{~nm}$ to1100nm can be considered safe for medical applications. Along the side the advancement in plastic optical fiber fabrication with accuracy to nano meter scale, biocompatibility, real-time signaling, low cost could not be overlooked, and so the use of optical fiber with light as interrogating agent, in medical device and probe applications presents as a better approach.

The main idea behind this research is to bring many optical fiber sensors on board to a single probe, for basic analysis of any inaccessible and highly localized area, where concentration and metabolism of the area under investigation can be analyzed, for the initial start the most important parameter, is to know the actual amount of $\mathrm{O}_{2}$ present under normal living conditions. This can be easily done by many devices already available in the market, but are not comfortable for long term use and it's impossible to have a very local analysis, at cellular level without disturbing the surrounding environment to have a actual processes undergoing inspection, it is quite difficult with regular devices, on the other hand with the help of optical fiber sensors this can be achieved with high precision and ease.

In addition Arduino UNO R3 is used; because of its open source nature which motivates developers to develop different programs. So combination of light, plastic optical fiber and simple processing units are integrated. The necessity of developing countries, for cheap diagnosis in healthcare is kept in focus. This project is developed with plastic multimode optical fiber, simple LED \& detectors and Arduino as controller board.

\section{BACKGROUND}

Optical fiber sensor diagram of Single fiber and double fiber medical arrangement is given below in figure 1 .

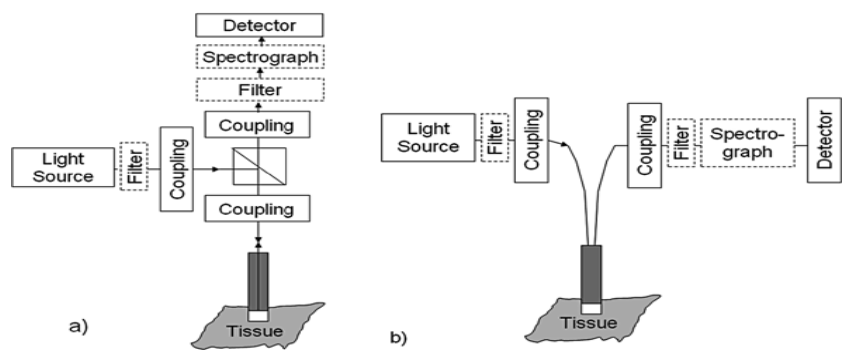

FIGURE 1. Typical optical fiber sensors arrangement

(a) a single fiber for both delivery and detection or

(b) separate source-detector fibers (after Utzinger and Richards-Kortum ).

Furthermore, optical fibres present very low attenuation in short distances; another property is the absence of crosstalk between fibres, which provides housing different sensors in the same catheter. In some cases a single electro-optic unit can be utilized for all the sensors, to provide required illumination of area, detection and signal-processing scheme.

An overview of fibre optic sensors for biomedical applications is given in section below, with particular attention to the sensors developed for in vivo sensing, advantages that these sensing are able to offer in different areas of application such 
as imaging, angiology, PPG, cardiovascular, intensive care, dermatology, gastroenterology, oncology, neurology, dentistry, ophthalmology etc. Also long range multi-spectral detectors are used, on which spectral analysis is performed in order to know the state of health of a particular gland, cell, tissue or cavity at different wavelength.

\subsection{Classification of Optical fiber Sensors for Biomedical}

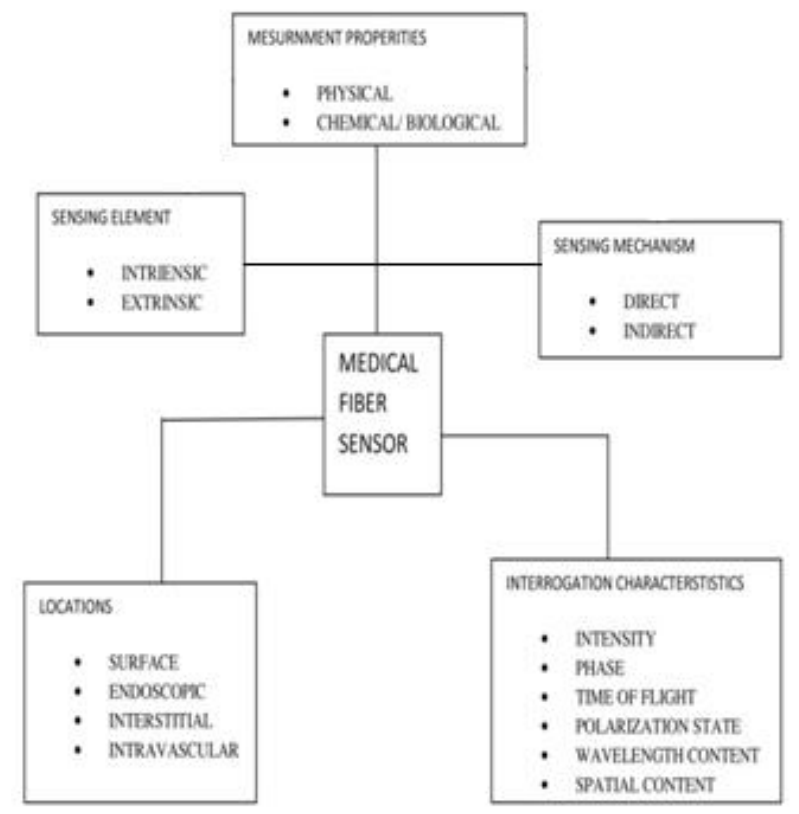

\section{MATERIALS \& COMPONENTS}

The system which is designed has an overall purpose of heart rate measurement of human using optical fiber as probe, which are of importance on the view of its application in biomedical. The project consists of simple configuration including the following components.

3.1 Arduino UNO processor unit which is an open source platform used by modern day developers for easy and cost effective implementation of many operations, in addition it has 6 analog I/O pins. The Uno has a $16 \mathrm{MHz}$ of clock inbuilt with an AT Mega 328 with $328 \mathrm{~kb}$ of memory sufficient for low data sensing devices. The platform also has 14 digital pin modes which can be used as digital input/ output, in our case as for different wavelengths of light LED attached to the pins. The six analog inputs are capable of processing 6 analog signals at same time. The Uno is also build with $+3.3 \mathrm{v},+5 \mathrm{v},+9 \mathrm{v}$ power supply and the ground given to both the analog and digital side of the platform. The most interesting part of the platform is that it can be connected to the computer for the real time display on the monitor, with a baud rate of 9600 to 115200 baud per second. Which is transferred to computer through a serial RS232 cable or WI-Fi module. The IDE used to operate the UNO platform is $4.5 \mathrm{MB}$ and requires a serial driver to drive the UNO through computer, the programs are compiled in the IDE verified and then can be uploaded in the UNO Platform with using a Port COM4. The serial monitor displays the real time values through a separate window.
3.2 Optical fiber: Being able to carry light is the property of optical fiber that it is considered an interesting asset in biomedical applications. Over that the quality of being immune to the surrounding, biocompatible, high speed transmission, no crosstalk's, real time, as desired for biomedical applications. The optical fiber that are used are plastic optical fiber of different combinations with two $1 \mathrm{~mm}$. and two $0.5 \mathrm{~mm}$ combination, also the optical fibers used are short distance (1-5 meters), and multimode fibers are considered. There is a greater scope of sensitivity and therapeutic application with a better tip design of the fiber which can be improved for the access of different types of biological parameters and with the advancement of the sensitivity of sensing gels or films are used on the probe tip. The integration of optical fiber provides continuous monitoring and it is also possible to monitor at nano meter scale.

3.3 Amplifier: The amplifier used is LM386 which is a audio analog signal amplifier which was suitable for the project application. The LM 386 has 8 - pin, with 1 and 8 being gain pins. Pin 2 is non-inverting (-), pin 3 is inverting $(+)$, pin 4 is grounded, pin 5 is the output pin, pin 6 is the power supply for the amplifier from $5 \mathrm{v}$ to $24 \mathrm{v}$. pin 7 is bypassed in the amplifier. There is a 10uf electrolyte capacitor to increase the gain of the amplifier which can be enhanced to 100 uf according to ratings of the amplifier. In our project the $5 \mathrm{v}$ power supply is provided to the amplifier.

3.4 Filters: used in the project shown in figure 2 is designed with taking heart rate in mind thus with the ideal heart beat of 60- 85 pulses per minute in an human, a low pass filter with the corner frequency of $16 \mathrm{~Hz}$. The resistor and the capacitor used is $100 \mathrm{~K} \mathrm{ohm}$ and $100 \mathrm{uF}$ respectively, which provides a suitable low pass for the frequencies for heart rate and rejects the frequencies above $16 \mathrm{~Hz}$. The filter can further be designed according to specific frequency analysis from the high frequencies to low frequencies.

\section{SIGNAL LOWPASS FILTER}

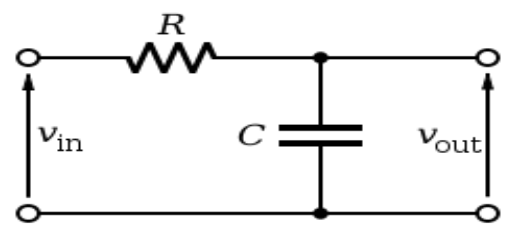

FIGURE 2. Filter circuit

$$
\begin{aligned}
& \mathrm{R}=100 \mathrm{Kohm} \\
& \mathrm{C}=100 \mathrm{uf} .
\end{aligned}
$$

We have

$$
\begin{aligned}
\frac{V_{0}}{V_{i}} & =\frac{1}{1+S R C} \\
& \frac{1}{1+S * 100 * 10^{3} * 100 * 10^{-6}}
\end{aligned}
$$




$$
\mathrm{H}(\mathrm{jw})=\frac{V_{0}(j w)}{V_{i}(j w)}=\frac{1}{1+j w 10}
$$

Angle $H(j w)=-\tan ^{-1}(10 w)$.

Thus act as low pass filter with cut-off frequency of $16 \mathrm{~Hz}$.

$\mathrm{f}_{\mathrm{H}}=\frac{1}{2 \pi * R * C}=\frac{1}{20 \pi}$

Low pass

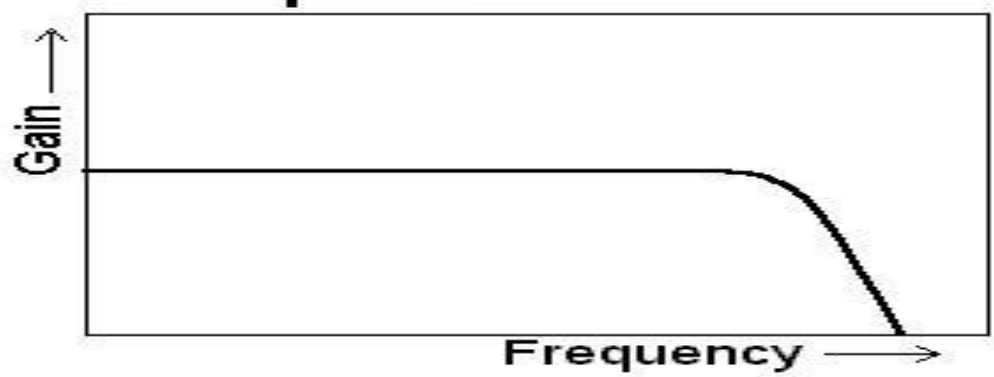

Fig 3: Low pass filter with cut-off frequency of $16 \mathrm{~Hz}$.

\section{BASIC SYSTEM DESIGN}

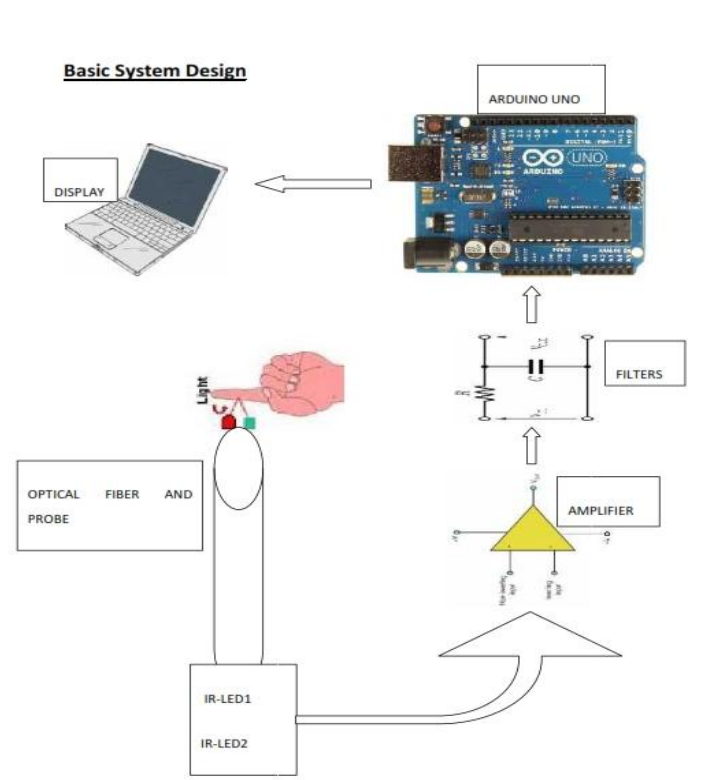

3.5 Light sources: Since light is the most useful for detection, and also the most harmless to human. The LED used for the project has a range of wavelength from $650 \mathrm{~nm}$ $940 \mathrm{~nm}$, with the arrangement as shown in circuit diagram. Holding the optical fiber together in an cylindrical hard plastic tubes and are insulated with electric tape.

3.6 Detector The photo diode used to measure the reflection of the light is a black epoxy infrared diode which has a sensing range from $850 \mathrm{~nm}-1000 \mathrm{~nm}$, with peak sensitivity of $940 \mathrm{~nm}$

\section{CIRCUIT DIAGRAM}

CIRCUIT DIAGRAM

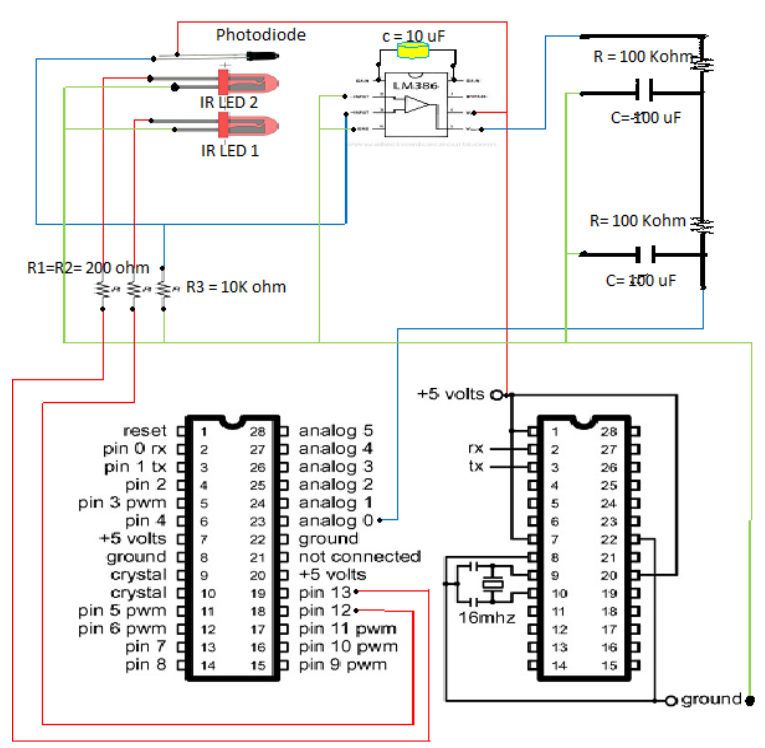




\section{SOFTWARE (Arduino IDE)}

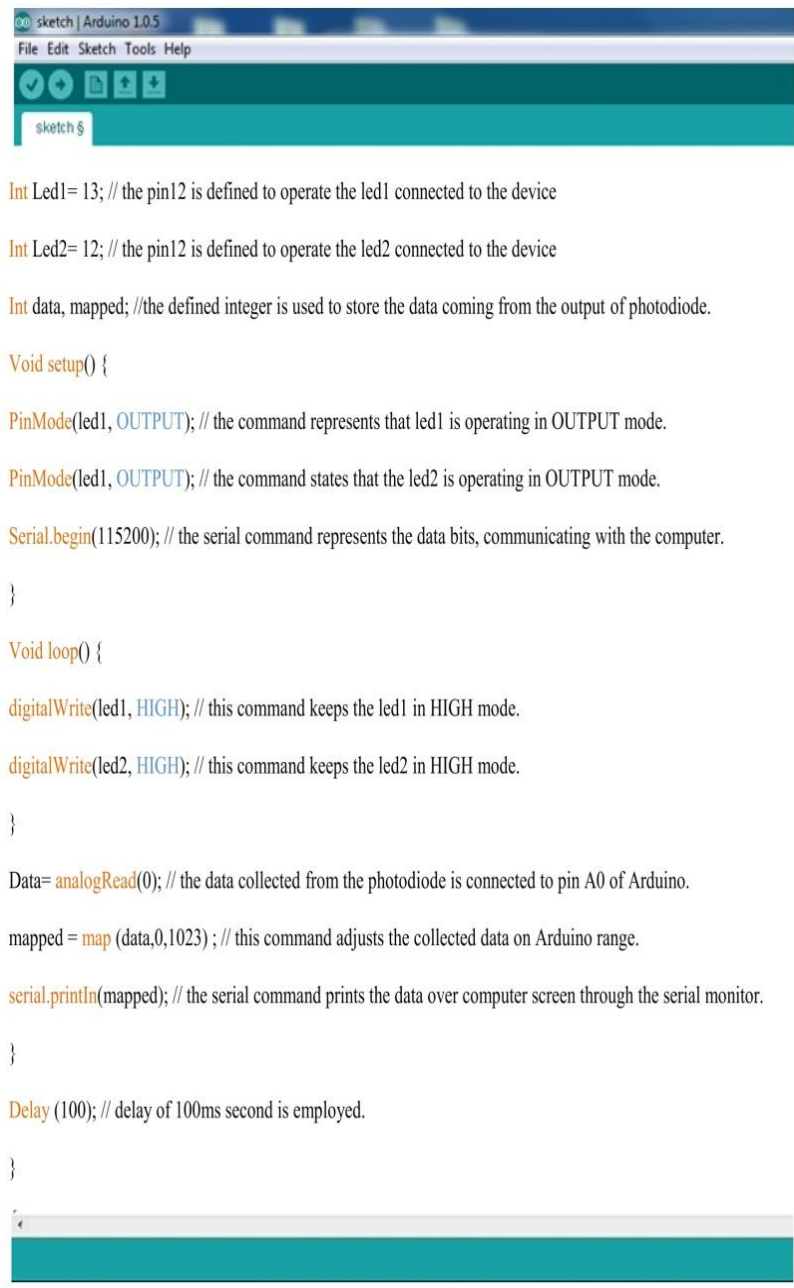

\section{WORKING \& ARRANGMENT}

The basic system design is the integration of optical fiber, IR Led and the supporting Arduino Uno hardware, which are connected to the computer through a RS-232 cable.

The system consists of Arduino microcontroller with ATMega 328 microcontroller chip. It has a power supply of $5 \mathrm{v}$, through which the LED are connected in a pair. The LED are connected to pin 12 and 13 set as digital output pins. And the photodiode with its negative side connected to the power supply of $+5 \mathrm{v}$ to through Arduino with a resistor of $10 \mathrm{k} \mathrm{ohm}$ between the positive end to ground is given to the analog input $\mathrm{A} 0$ of the Arduino Uno.

The probe consist of four optical fiber with two $1 \mathrm{~mm}$ and two $0.5 \mathrm{~mm}$ core bundle. The fibers are divided into 2 sets for propagation of light through them. At the tip of the probe the photodiode is placed to collect the reflected light from the sample. All the fibers and the photodiode are mounted together with a clip to hold the investigation area.

The amplifiers and filters are connected to quantify precision of the incoming signals to Arduino Uno. The filters are used to reduce the noise and provide smoothness to the incoming signal at A0, with a cutoff frequency of $16 \mathrm{HZ}$.
The software provided in above software section. Provides logical loops for the pin high mode $(12,13)$ which are connected to the led in the arrangement, the samples are taken per $100 \mathrm{~ms}$ and loaded through analog input at A0 to store in the variable defined as [Mapped]. Then the mapped is displayed at the Arduino serial monitor with 115200 baud rate for processing purposes. The obtained values are displayed in the digital range of 0 to 1023 . This provides high range of sensitivity with 1023 levels of values in the display.

The values obtained then be used to calibrate heart rate with the help of Microsoft excel, using the values graph is plotted. With reflection pr unit area vs. time in seconds

\section{OBSERVATIONS TABLE}

Procedure and values obtained from heart rate measurement

The probe with optical fiber and photodiode is placed on any one area of body where the flow of blood is very large and the blood vessels are easily accessible through the skin barrier. The readings are taken per $100 \mathrm{~ms}$ with the optical fiber probe.

Step.1. The values obtained are taken at $100 \mathrm{~ms}$ for 64 seconds are as following.

Values obtained for $64 \mathrm{sec}$. with sample time of $100 \mathrm{~ms}$. Plotted A and B. Shown in table 1.

TABLE-1

\begin{tabular}{|c|c|c|c|c|c|}
\hline 4 & A & B & 4 & A & B \\
\hline 1 & 100 & 214 & 626 & 62600 & 303 \\
\hline 2 & 200 & 327 & 627 & 62700 & 306 \\
\hline 3 & 300 & 324 & 628 & 62800 & 307 \\
\hline 4 & 400 & 324 & 629 & 62900 & 309 \\
\hline 5 & 500 & 323 & 630 & 63000 & 309 \\
\hline 6 & 600 & 321 & 631 & 63100 & 308 \\
\hline 7 & 700 & 321 & 632 & 63200 & 306 \\
\hline 8 & 800 & 321 & 633 & 63300 & 308 \\
\hline 9 & 900 & 319 & 634 & 63400 & 307 \\
\hline 10 & 1000 & 320 & 635 & 63500 & 307 \\
\hline 11 & 1100 & 319 & 636 & 63600 & 308 \\
\hline 12 & 1200 & 320 & 637 & 63700 & 306 \\
\hline 13 & 1300 & 319 & 638 & 63800 & 305 \\
\hline 14 & 1400 & 318 & 639 & 63900 & 307 \\
\hline 15 & 1500 & 319 & 640 & 64000 & 307 \\
\hline 16 & 1600 & 319 & 641 & 64100 & 308 \\
\hline 17 & 1700 & 319 & 642 & 64200 & 307 \\
\hline 18 & 1800 & 318 & 643 & 64300 & 304 \\
\hline 19 & 1900 & 319 & 644 & 64400 & 306 \\
\hline 20 & 2000 & 318 & 645 & 64500 & 307 \\
\hline 21 & 2100 & 317 & 646 & & \\
\hline 22 & 2200 & 318 & 647 & & \\
\hline 23 & 2300 & 317 & 648 & & \\
\hline 24 & 2400 & 317 & 649 & & \\
\hline 25 & 2500 & 316 & 650 & & \\
\hline
\end{tabular}


Step.2

The average of 645 values that estimates to 64.5 seconds is taken and averaged to find the peak varation value from the table which is found to be as follows in table 2 .

AVERAGE OF 645 VALUES $=309.6527 .=\mathrm{C} 1$

TABLE-2

\begin{tabular}{|c|c|c|c|c|c|c|}
\hline \multicolumn{3}{|c|}{$\mathrm{C} 1$} & \multicolumn{2}{|c|}{$f_{x}$} & \multicolumn{2}{|c|}{$=$ AVERAGE (B1:B645) } \\
\hline 4 & A & B & $\mathrm{C}$ & D & $E$ & $\mathrm{~F}$ \\
\hline 1 & 100 & 214 & 309.6527 & & & \\
\hline 2 & 200 & 327 & & & & \\
\hline 3 & 300 & 324 & & & & \\
\hline
\end{tabular}

\section{Step.3}

In this table all the 645 values are subtracted from the average so to obtain the maximum and minimum values in the table and multiply by 1000 to add gain, given in table 3 .

Average subtracted $=(\mathrm{B} 1-309.6527) * 1000 .=\mathrm{D}: 1: 645$

TABLE-3

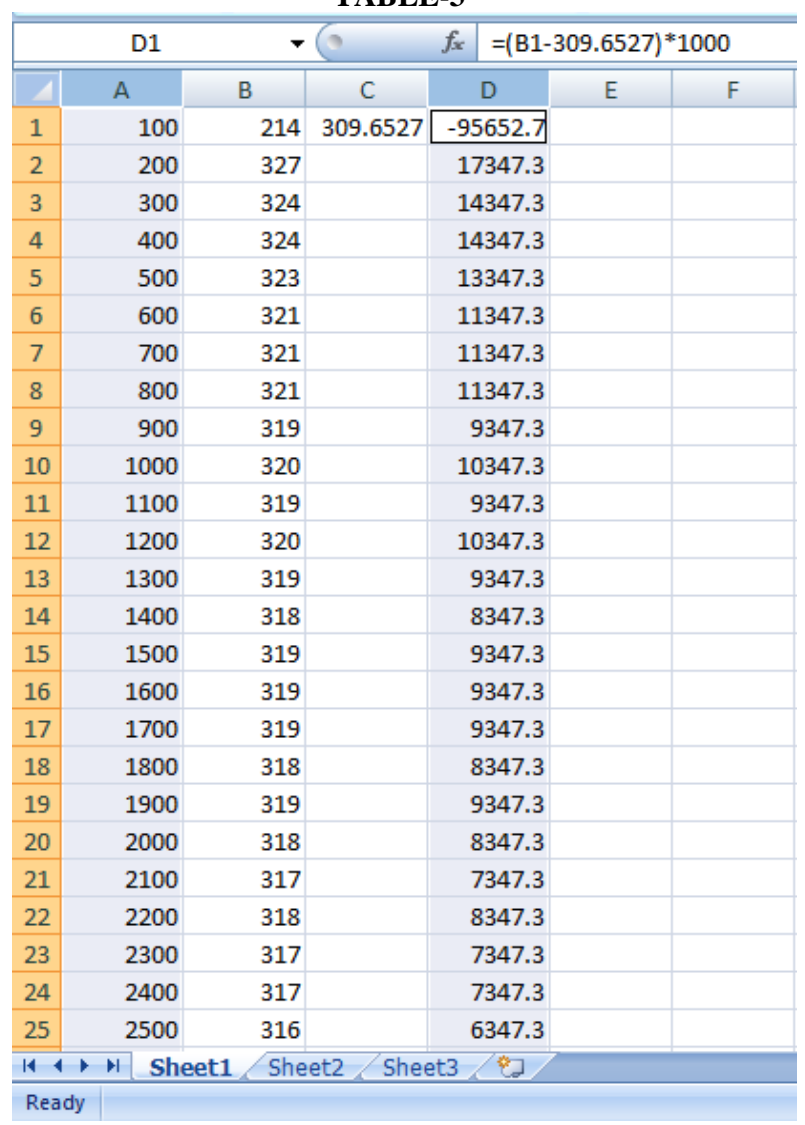

Step 4.

The clear peak values is selected from the table and plotted with respect to time in the given table, the table is shown and the graph of $4000 \mathrm{~ms}$ is displayed with $0.8 \mathrm{sec}$ interval between consequent heart pulses, shown in table 4 .

Sample values for $4000 \mathrm{~ms}$. Plotted in E:F
TABLE-4

\begin{tabular}{|c|c|c|c|c|c|c|c|c|}
\hline \multicolumn{3}{|c|}{$\mathrm{F} 1$} & (3) $f_{x}$ & \multicolumn{2}{|c|}{\begin{tabular}{l|l}
$x$ & 2347.30000000002
\end{tabular}} & \multirow[b]{2}{*}{$\mathrm{F}$} & \multirow[b]{2}{*}{ G } & \multirow[b]{2}{*}{ H } \\
\hline 4 & A & B & C & D & E & & & \\
\hline 1 & 100 & 214 & 309.6527 & -95652.7 & 100 & 2347.3 & & \\
\hline 2 & 200 & 327 & & 17347.3 & 200 & 3347.3 & & \\
\hline 3 & 300 & 324 & & 14347.3 & 300 & 2347.3 & & \\
\hline 4 & 400 & 324 & & 14347.3 & 400 & 3347.3 & & \\
\hline 5 & 500 & 323 & & 13347.3 & 500 & 3347.3 & & \\
\hline 6 & 600 & 321 & & 11347.3 & 600 & 2347.3 & & \\
\hline 7 & 700 & 321 & & 11347.3 & 700 & 1347.3 & & \\
\hline 8 & 800 & 321 & & 11347.3 & 800 & 2347.3 & & \\
\hline 9 & 900 & 319 & & 9347.3 & 900 & 3347.3 & & \\
\hline 10 & 1000 & 320 & & 10347.3 & 1000 & 2347.3 & & \\
\hline 11 & 1100 & 319 & & 9347.3 & 1100 & 2347.3 & & \\
\hline 12 & 1200 & 320 & & 10347.3 & 1200 & 1347.3 & & \\
\hline 13 & 1300 & 319 & & 9347.3 & 1300 & 14347.3 & & \\
\hline 14 & 1400 & 318 & & 8347.3 & 1400 & 2347.3 & & \\
\hline 15 & 1500 & 319 & & 9347.3 & 1500 & 2347.3 & & \\
\hline 16 & 1600 & 319 & & 9347.3 & 1600 & 3347.3 & & \\
\hline 17 & 1700 & 319 & & 9347.3 & 1700 & 3347.3 & & \\
\hline 18 & 1800 & 318 & & 8347.3 & 1800 & 347.3 & & \\
\hline 19 & 1900 & 319 & & 9347.3 & 1900 & 2347.3 & & \\
\hline 20 & 2000 & 318 & & 8347.3 & 2000 & 1347.3 & & \\
\hline 21 & 2100 & 317 & & 7347.3 & 2100 & 3347.3 & & \\
\hline 22 & 2200 & 318 & & 8347.3 & 2200 & 2347.3 & & \\
\hline 23 & 2300 & 317 & & 7347.3 & 2300 & 1347.3 & & \\
\hline 24 & 2400 & 317 & & 7347.3 & 2400 & 2347.3 & & \\
\hline 25 & 2500 & 316 & & 6347.3 & 2500 & 2347.3 & & \\
\hline
\end{tabular}

\section{Graph 1.}

Graph 1 shows the interval between heart rate from 0 to $3.5 \mathrm{sec}$.

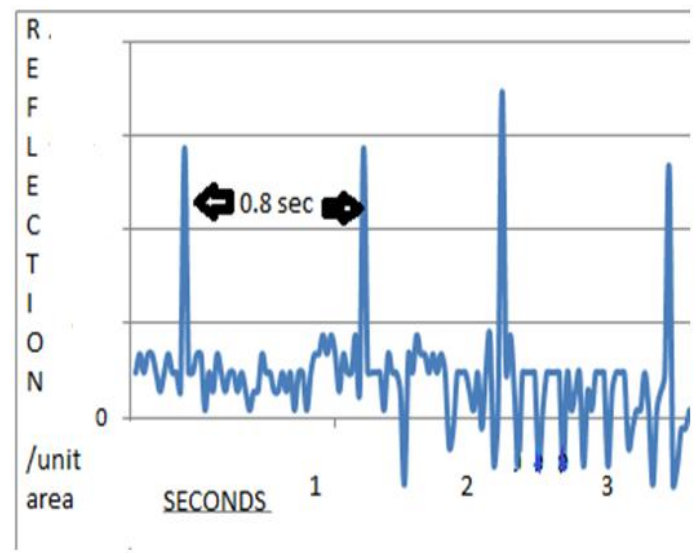

The graph represented displays the pulse rate of the human heart for $4000 \mathrm{~ms}$, the approximate distance between the two intervals is shown as being $0.8 \mathrm{sec}$.

Step 5. The value for $1200 \mathrm{~ms}$ is again selected and displayed in the table and plotted in the Graph shown.

Sample values for 1200 ms. Column Plotted G1:H1.

Average PULSE Rate Results to be $0.75-0.85$. 
TABLE-5

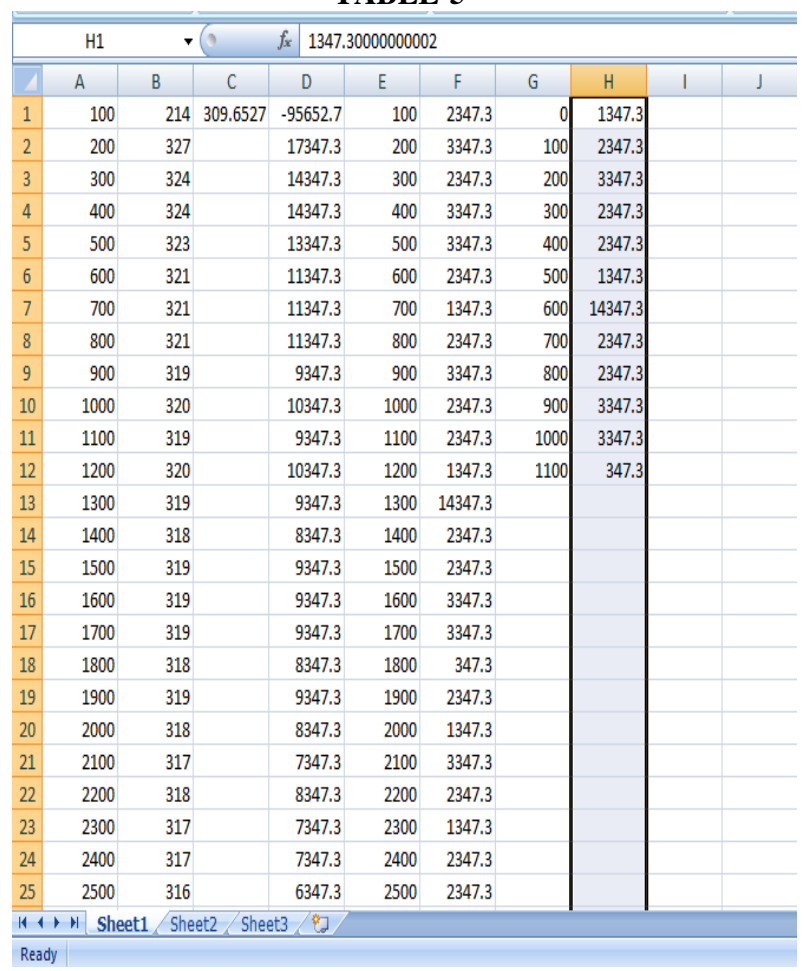

Graph 2: shown for pulse between 0 - 1200ms

Which shows that the pulse duration detected by the device using optical fiber is given by $0.8-0.75$ per beat. This is then calibrated with the ECG given in the graph shown below which also concludes that the pulse is almost rightly detected by the device.

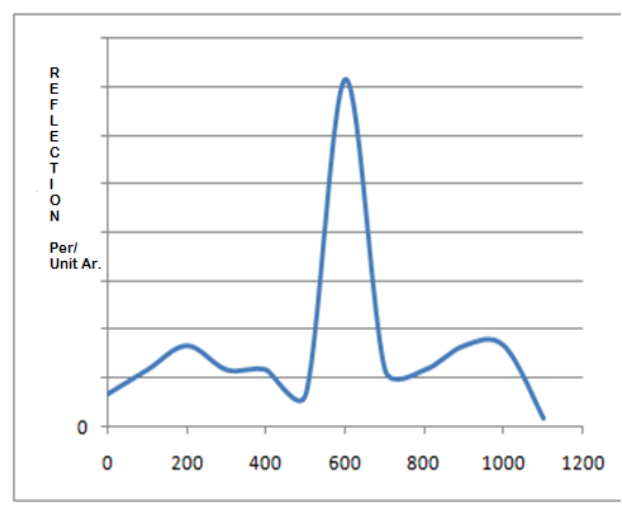

Graph-2

\section{COMPARISON OF RESULT WITH ECG WAVEFORM}

The ECG wave form is taken from (http://howmed.net/physiology/electrocardiogram-ecg/).

Which describes the wave duration and the pulse rate of the human heart. The derived pulses are as follows.

P-R- Interval : 0.12- $0.20 \mathrm{sec}$

QRS Interval: 0.08- $0.10 \mathrm{sec}$.

Q-T Interval : $0.4-0.43 \mathrm{sec}$.

R-R Interval : 0.6- $1.0 \mathrm{sec}$.

\section{GRAPH-3 ECG Waveform}

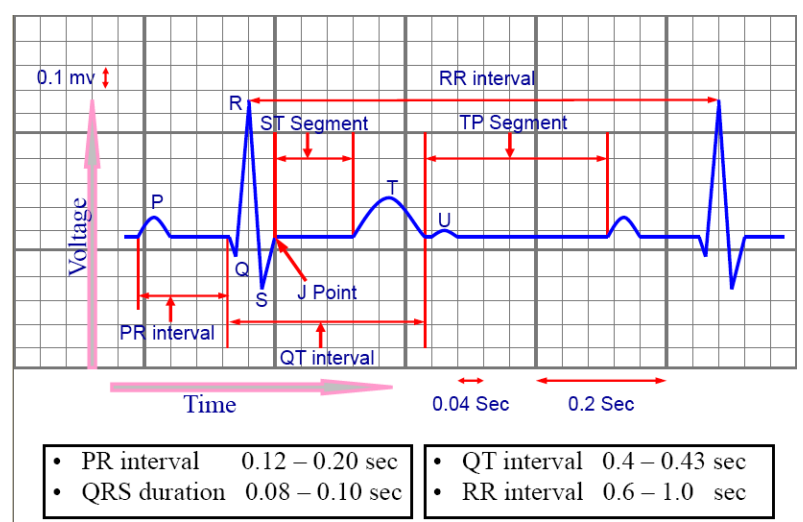

\section{CONCLUSION}

With this dissertation we intended to design the optical fiber based heart rate sensor, the PPG pulses obtained from our designed device is accurate, when compared with the existing ECG signals, shows the exact time duration of one heart beat to be $0.65-0.8$ seconds between R-R intervals as that of ECG. The Arduino Uno used for processing the incoming signals with six analog inputs proves to have high potential for simple and low cost analysis. This device can be extensively used in hospitals, contributing towards the health of our country. As the capacity of the optical fiber gets larger and the cost of fiber decreases progressively there is more and more scope for the development of optical fiber design and applications in biomedical areas.

\section{REFERENCES}

[1] D.K. Harrison*, P.T. Mccollum**, D.J. Newton*, p. Hickman** and A.S. Jain***.1994. Amputation level assessment using lightguide spectrophotometry. Prosthetics and Orthotics International, 1994, 19, 139147

[2] L. Asare, E. Kviesis-kipge, M. Ozols, J. Spigulis, and R. Erts.(2012). Multi-spectral optoelectronic device for skin microcirculation analysis. Lithuanian Journal of Physics, vol. 52, no. 1, pp. 59-62 (2012).

[3] M. Veronica Rigo and Peter Geissinger. 2012. Crossed optical fiber sensor arrays for high-spatial-resolution sensing: application to dissolved oxygen concentration measurements. Hindawi Publishing Corporation Journal of Sensors volume 2012, article id 464092, 10 pages doi:10.1155/2012/464092.

[4] A. Cysewska-sobusiak. 2002. Noninvasive optoelectronic monitoring of the living tissues vitality. third International Workshop on Robot Motion and Control, November 9-1 1, 2002.

[5] Janis Spigulis, Lasma Gailite, Alexey Lihachev, and Renars Rts. 2007 Simultaneous recording of skin blood pulsations at different vascular depths by multiwavelength photoplethysmography. (C) 2007 Optical Society of America Ocis codes: 170.4580, 170.0170. 
[6] Vicky kumar Singh, Sumit kumar Thakur, Vinod kumar. 2014.Application of heart PPG. International Journal of Electrical, Electronics and Data Communication, issn: 2320-2084 volume-2, issue-3, march-2014.

[7] V. A. Korolyov* and V. T. Potapov. 2011 Biomedical Fiber_Optic Temperature and Pressure Sensors. Biomedical Engineering, vol. 46, no. 2, july, 2012, pp. 79_82. translated from meditsinskaya tekhnika, vol. 46, no. 2, mar._apr., 2012, pp. 38_42.original article submitted april 4, 2011.

[8] Juneyoung Kim. 2013. Design of infrared sensor based measurement system for continuous blood pressure monitoring device. Thesis submission university of Minnesota. U.S.
[9] Wesley Nguyen and Ryan Horjus. 2011. Heart-rate monitoring control system using photoplethysmography (ppg). electrical Engineering Department California Polytechnic State University, San Luis Obispo.

[10] Tsukasa Funane, Hirokazu Atsumori, Masashi Kiguchi, Yukari Tanikawa, and Eiji Okada. (2011); Optical scanning system for light-absorption measurement of deep biological tissue. citation: rev. sci. instrum. 82, 093101 (2011); doi: 10.1063/1.3632133.

[11] L. Fodor et al. 2011 light tissue interaction. aesthetic applications of intense pulsed light, doi: 10.1007/978-184996-456-2_2, () Springer-Verlag London limited. 